\title{
Collective Choice with Endogenous Reference Outcome*
}

\author{
Hannu Vartiainen \\ Yrjö Jahnsson Foundation ${ }^{\dagger}$ \\ University of Helsinki, Department of Economics \\ Discussion Papers 597:2004 \\ ISSN 1459-3696 \\ ISBN 952-10-1527-6
}

\begin{abstract}
A collective choice problem - essentially a bargaining problem without disagreement outcome - is studied. An exteneded solution, which determines simultaneously a solution and a reference point, is characterized through a system of axioms. It is proved that the unique extended solution meeting Pareto-optimality, independence of irrelevant alternatives, symmetry, and scale invariance axioms maximizes the Nash product via both the solution and the reference point.

JEL: Cr1, Cr78.

Keywords: No disagreement, Bargaining theory, Axioms.
\end{abstract}

\section{Introduction}

We study collective choice situation where two players need to choose from the set of joint actions. We assume that only the induced utilities affect the choice, and hence concentrate on the utility representation of the problem. The utility possibility set, which we assume to be compact and convex set in $\mathbb{R}^{2}$, is the only primitive of our model. Thus the central difference between our model and the bargaining problem á la Nash (1950) is that we do not assume the existence of an exogenously given disagreement outcome.

In many scenarios, a disagreement outcome can be determined on natural grounds. In such cases bargaining theory provides the natural framework to analyse collective decision making. However, in other scenarios neither

\footnotetext{
${ }^{*}$ I am grateful to an associate editor and a referee, whose constructive criticism improved the paper significantly. I also thank Klaus Kultti and Hannu Salonen for benefical comments, and Ehud Kalai for guidance at the early stages of the project.

${ }^{\dagger}$ Ludviginkatu 3, FIN-00130 Helsinki. E-mail: hannu.vartiainen@yjs.fi.
} 
the existence nor placing of the disagreement outcome is clear at the outset, and they need to be assumed on ad hoc grounds. A theory which does not rely on exogenous disagreement outcome, or which generates a disagreement outcome endogenously, would be highly helpful in such situations. Coming up with such theory is the aim of this paper. ${ }^{1}$

We departure from the standard bargaining framework by how we model the solution. Collective choice is analyzed through an extended solution $F$ that consists of pairs of utility vectors and may, a priori, have multiple values. Specifically, under collective choice problem $U$, extended solution $F(U)$ is a nonempty convex set in $U \times U$. Thus an element of the extended solution $F(U)$, say $(s, r)$, is an ordered pair of utility vectors. The first entry, " $s "$, of such ordered pair is called as a solution and the second entry, " $r$ ", as a reference outcome.

Heuristically, the role of a reference outcome is to motivate the solution through the standard counterfactual argument: Solution $s$ constitutes an acceptable compromise given that in the absence of cooperation players would end up choosing $r .^{2}$ Of course, the question is not only how to choose a solution given the reference outcome, but also how to choose a reference outcome given the solution it induces. A priori, the two selection problems seem redundant. Since we are primarily interested in the solution, why not simply disregard the reference outcome altogether from the definition of the problem, and focus solely on the solution? The point is that a reference outcome allows a point from which utility comparisons can be made. Thus with a reference outcome the problem of choosing the solution as a function of preferences can be translated into one of choosing the solution on the basis of utility comparisons. As pointed out by Conley at. al. (1997, forth.) and Thomson (1981), this difference is crucial. It allows one to circumvent the impossibility problems familiar in the social choice literature. This issue is further discussed in the final section of the paper.

In practice, the key problem with a bargaining problem without a reference point is that the standard axiomatizations are not powerful enough to pin down a well defined solution. ${ }^{3}$ The point of this paper is to show that this problem can be circumvented simply by assuming that the choices of both the solution and the reference point are governed by the same princi-

\footnotetext{
${ }^{1}$ Roth (1977), Thomson (1981), and Conley at.al (1997, forth.) replace a fixed disagreement point by a reference function that map each utility possibility set to a point (not necessarily in the utility possibility set) in $\mathbb{R}^{2}$. To induce a well specified solution, such function needs to satisfy certain conditions. However, many functions meet these conditions and, in general, many classes of solutuons may be indiuced. There is no immediate rationale to rank one reference function over another.

${ }^{2}$ This interpretation is attractive especially if $U$ is induced by correlated equilibria of a noncooperative game.

${ }^{3}$ Conley at.al. (2000) (see also Sen, 1970, and Myerson, 1978) show that no social choice rule satisfies the natural counterparts of the axioms that characterize the standard bargaining solutions.
} 
ples. Simultaneous choice of the solution and the reference point provides enough discipline to carry out the analysis in the standard fashion.

The axioms we impose on the extended solution are directly analogous to those by Nash (1950). As there is no fixed disagreement point, and the extended solution may be multivalued, the axioms must be adjusted accordingly. Let $U$ and $V$ be choice problems.

- Pareto-optimality: $s$ is Pareto-optimal for all elements $(s, r)$ of $F(U)$.

- Symmetry: if $U$ is a symmetric problem, then $F(U)$ contains a playerwisely symmetric element.

- Independence of Irrelevant Alternatives (IIA): if $U$ is contained by $V$, and $(s, r)$ is contained by $F(V)$ and $U \times U$, then $(s, r)$ is contained by $F(U)$.

- Scale Invariance: $F$ is invariant to utility scales.

The rationale for Pareto-optimality, scale invariance, and symmetry axioms (that we call the "extended axioms") are analogous to those discussed by Nash (1950). The IIA axiom is slightly more demanding since now the consistency principle behind IIA implicitly assumes that the reference point selection process is governed by some kind of optimality considerations, too.

Our Main Theorem is stated as follows: In the domain of compact and convex utility possibility sets, extended solution $F$ satisfies Paretooptimality, symmetry, IIA and scale invariance axioms if and only if $F=F^{*}$, where

$$
F^{*}(U)=\arg \max _{u, v \in U}\left(u_{1}-v_{1}\right)\left(u_{2}-v_{2}\right), \quad \text { for any problem } U .
$$

Solution $F^{*}$ is called as the extended Nash solution. Note that $F^{*}$ is single valued whenever $U$ is strictly convex.

The key feature of the extended Nash solution is that it does not require the existence of a predefined disagreement point. To the contrary, the reference point is determined endogenously by the property that $(s, r) \in F^{*}(U)$ if and only if $s$ is the standard Nash solution of bargaining problem $(U, r)$, and $-r$ is the standard Nash solution of bargaining problem $(-U,-s)$. Thus $(s, r) \in F(U)$ and $(-r,-s) \in F(-U)$ constitute one another's "dual" solutions.

As usual, sufficiency is easy. The proof of the necessary part is based on three key insights, which we now sketch. First, due to the above described "duality" property of $(s, r) \in F^{*}(U)$, points $s$ and $r$ lie in the boundary of $U$, and the slope of the tangents of the boundary that contain these points is in both cases equal to $\left(s_{2}-r_{2}\right)\left(s_{1}-r_{1}\right)^{-1}$. One should note that only the elements of $F^{*}(U)$ have this property. Now take any $\left(s^{\prime} r^{\prime}\right) \in F(U)$. 
Assuming that $r^{\prime}$ lies in the boundary we can proceed along the standard avenue (symmetric rectangle that touches scaled $U$ at $(1,1)$ and $(-1,-1)$ ) to prove that $(s, r) \in F(U)$ for any $F$ meeting the four axioms.

The remaining problem is then to show that $r^{\prime}$ must lie in the boundary. To prove this, we first show that in the domain of strictly convex and smooth problems, when $F^{*}$ is single valued, this indeed is the case. The reason for this is that if $r^{\prime}$ were not in the boundary (recall that $s^{\prime}$ lies in the Pareto frontier), then there is a large degree of freedom to scale $U$ in a way the the scaled version of $U$ contains $\left(s^{\prime}, r^{\prime}\right)$ and is still contained by $U$. By IIA, the extended solutions of each scaled utility set induces the same extended solution. But this is in contradiction with the scale invariance axiom. Thus $r^{\prime}$ has to lie in the boundary. This in turn implies that in the domain of strictly convex and smooth problems $F$ meets the axioms if and only if it is equivalent to $F^{*}$.

Finally, to prove the result in the general domain, note that for any symmetric and convex problem $U$ there is symmetric, smooth and strictly convex problem $E$ (e.g. a $45^{\circ}$-inclined ellipse) that is contained by $U$ and has the property that $F^{*}(E) \in U$. As the previous paragraph implies, strict convexity and smoothness of $E$ imply that $F(E)$ coincides $F^{*}(E)$. But this means that $F(U)$ has to be equal to $F^{*}(U)$ since otherwise IIA would imply that $F(E)$ contains another element, too. Thus $F$ is equivalent to $F^{*}$ also in the unrestricted domain.

A Generalized Framework However, the formulation of the extended solution leaves open some intepretational problems. Why is it that the selected outcome is dependent on a reference outcome anyway? And why is it that it dependent on only a single reference outcome rather than a set of reference points? To answer these questions, consider the following story on how players negotiate.

At the outset, choice problem would be solved if players would be able to form a collective preference ordering on $U$ : the top ranked element in the ordering would constitute the optimal choice. Thus the problem is of agreeing on collective preferences. The key to solve this problem is to note that reducing the set of alternatives must make it less difficult to come up with collective preferences. Thus to reduce the difficulty of forming collective preferences, we may assume that players bargain by eliminating outcomes that are known to be collectively preferred by some other alternative. The elimination process is continued until a set of alternatives, say $G \subseteq U$, is reached on which a collective preference ordering, say $\succ$, can be defined. The collective choice $s$ is now defined by the property $s:=\max _{\succ} G{ }^{4}$

To relate this story to the extended solution $F$, one let each element $(s, r) \in F(U)$ define a binary reference set $G=\{s, r\}$ and preference or-

\footnotetext{
${ }^{4}$ Existence of such point is assumed.
} 
dering $s \succ r$. Thus $F$ could reflect the endogenous collective choice process as described above. However, nothing in the framework forces one to concentrate only on this family of reference sets. An interesting result is that under appropriate axiomatic constraints this indeed is the case.

To prove this claim, define a generalized solution $\mathcal{G}$ that contains, under each $U$, a family of triples $(s, G, \succ)$, whose elements are as described in the story above. Next define the "generalized" versions of the above axioms on solution $\mathcal{G}$. The most notable changes in the axioms are related to symmetry and IIA: Solution $\mathcal{G}$ meets the generalized symmetry axiom if $\mathcal{G}(U)$ contains a symmetric element $(s, G, \succ)$ such that $G$ is symmetric and $\left(u_{1}, u_{2}\right) \succ$ $\left(v_{1}, v_{2}\right)$ implies $\left(u_{2}, u_{1}\right) \succ\left(v_{2}, v_{1}\right)$, for all $u, v \in G$, whenever $U$ is symmetric. Solution $\mathcal{G}$ meets the generalized IIA if $U \subseteq V,(s, G, \succ) \in \mathcal{G}(V)$, and $G \cap U$ contains at least two elements of which one is $s$, imply $(s, G \cap U, \succ) \in \mathcal{G}(U)$.

The result is that there is a unique generalized solution $\mathcal{G}^{*}$ that meets the generalized Pareto-optimality, symmetry, IIA, and scale invariance axioms. Such $\mathcal{G}^{*}$ is defined by the property

$$
\mathcal{G}^{*}(U):=\{(s,\{s, r\}, \succ):(s, r) \in F(U) \cap \succ\}, \text { for all } U \in \mathcal{U} .
$$

After noting that in a symmetric problem $U$ the reference set lies in the diagonal, the proof of this theorem is almost complete restatement of the proof of the Main Theorem. Thus $F^{*}$ can be seen as the reduced form expression of a the only reasonable generalized solution $\mathcal{G}^{*}$. This provides further motivation for the extended Nash solution.

Outline We begin with specifying the model and the axioms. Then we characterize the extended Nash solution and establish the results, first in the general domain with restricted reference point, and then in the restricted domain with unrestricted reference point. Finally we combine the arguments, and prove the Main Theorem. After this, we analyse the generalized solution and establish the characterization. The final section discusses related literature and provides some remarks.

\section{The Model}

Let

$$
\mathcal{U}=\left\{U \subset \mathbb{R}^{2}: U \text { compact and convex, and has nonempty interior }\right\}
$$

be the domain of utility possibility sets $U$. Mapping $F: \mathcal{U} \rightarrow \mathbb{R}^{2} \times \mathbb{R}^{2}$ is an extended solution correspondence if $F(U)$ is convex $^{5}, F(U) \subset U \times U$ and $(u, u) \notin F(U),{ }^{6}$ for all $u \in U \in \mathcal{U}$. For any ordered pair $(s, r) \in F(U)$,

\footnotetext{
${ }^{5}(u, v),\left(u^{\prime}, v^{\prime}\right) \in F(U)$ implies $\left(u \lambda+u^{\prime}(1+\lambda), v \lambda+v^{\prime}(1+\lambda)\right) \in F(U)$ for all $\lambda \in[0,1]$.

${ }^{6}$ This restriction is possible since $U$ is not singleton by assumption. I thank the associate editor for pointing this out.
} 
the first entry, $s$, is called as the solution and the second entry, $r$, as the reference point. The role of the reference point is to serve as a basis for the standard counterfactual argument: Solution $s$ is collectively chosen since otherwise players would collectively choose $r$, and $s$ is collectively preferred over $r$. The central question is how what is the right form of the extended solution.

The axioms we are interested the extended solution correspondence to satisfy are familiar from bargaining theory. They are directly analogous to those imposed by Nash (1951).

Use the notation $a U+b=\left\{\left(a_{1} u_{1}+b_{1}, a_{2} u_{2}+b_{2}\right):\left(u_{1}, u_{2}\right) \in U\right.$, $\left.\left(a_{1}, a_{2}\right),\left(b_{1}, b_{2}\right) \in \mathbb{R}^{2}\right\}$. When both coordinates of $u$ are multiplied by scalar $\lambda$, we write $\lambda u$. In particular, write $-1 U=-U$.

EINV (Extended Scale Invariance) $F(a U+b)=a F(U)+b$, for $a \in \mathbb{R}_{++}^{2}, b \in$ $\mathbb{R}^{2}$, for all $U \in \mathcal{U}$.

EIIA (Extended Independence of Irrelevant Alternatives) $(s, r) \in F(V) \cap$ $U \times U$ and $U \subseteq V$ implies $(s, r) \in F(U)$, for all $U, V \in \mathcal{U}$.

Motivation for the independence condition is analogous to the original one: If a particular utility comparison standard is "collectively optimal" in a larger utility domain, given the solution it induces, then, if the standard is feasible also in a smaller domain, it should also be optimal in this domain.

Denote the transpose of utility possibility set $U$ by $U^{\prime}:=\left\{\left(u_{2}, u_{1}\right) \in \mathbb{R}^{2}\right.$ : $\left.\left(u_{1}, u_{2}\right) \in U\right\} . U$ is symmetric if $U=U^{\prime}$.

ESYM (Extended Symmetry) $U=U^{\prime}$ implies $\left(s_{1}, r_{1}\right)=\left(s_{2}, r_{2}\right)$ for some $(s, r) \in F(U)$.

Due to its potential multi-valuedness, $F(U)$ need not be symmetric when $U$ is symmetric - $F(U)$ only needs to $a$ symmetric element. If $F(U)$ is single valued, then it is symmetric when $U$ is symmetric.

Denote the Pareto frontier by $P(U)=\{u \in U: v \geq u$ implies $v \notin U\} .{ }^{7}$

PAR (Pareto-optimality) $s \in P(U)$, for all $U \in \mathcal{U}$, for all $(s, r) \in F(U)$.

Note that Pareto-optimality implies that if $U$ is strictly convex, then $\{s:(s, r) \in F(U)\}$ is single valued.

\subsection{The Extended Nash}

Let the extended Nash solution correspondence $F^{*}: \mathcal{U} \rightarrow U \times U$ be defined by the following property:

$$
F^{*}(U)=\underset{(u, v) \in U \times U}{\arg \max }\left(u_{1}-v_{1}\right)\left(u_{2}-v_{2}\right) .
$$

\footnotetext{
${ }^{7}$ Vector inequalities: for $u, v \in \mathbb{R}^{2}, u \geqq v$ means $u-v \in \mathbb{R}_{+}^{2} ; u \geq v$ means $u \geqq v \neq u$; $u>v$ means $u-v \in \mathbb{R}_{++}^{2}$.
} 
The difference between the extended Nash solution and the standard Nash solution (to be discussed in more precise terms below) under $U$ is that the latter is defined with respect to an exogenously determined reference point $r$. In contrast, $F^{*}$ allows $r$ to be determined endogenously, and simultaneously with $s .^{8}$

The geometry of $F^{*}(U)$ will play influential role in the analysis. As the following parity manifests, $s$ and $r$, for $(s, r) \in F^{*}(U)$ have the interpretation of being one anothers" "dual" solutions:

$$
(s, r) \in F^{*}(U) \text { if and only if }(-r,-s) \in F^{*}(-U) .
$$

To see the geometry of $F^{*}(U)$, choose scales of $U$ such that $(s, r) \in F^{*}(U) \cap$ $(-U)$. Applying (2) twicely, we have $(-s,-r) \in F^{*}(U)$. Thus $S^{*}:=\{s$ : $\left.(s, r) \in F^{*}(U)\right\}$ and $D^{*}:=\left\{r:(s, r) \in F^{*}(U)\right\}$ constitute line segments of equal length and slope in the boundary of $U$, at the opposite sides of the origin. Thus the $\operatorname{co}\left\{S^{*}, D^{*}\right\}$ is a parallelogram whose diagonals run through the origin. This means that $F^{*}(U)$ is single valued whenever the boundary of $U$ does not have flat parts - i.e. when $U$ is strictly convex. It also follows that the boundary has at points $s$ and $r$ tangent with slope $\left(s_{2}-r_{2}\right)\left(s_{1}-r_{1}\right)^{-1}$.

For future reference, identify function $\left(s^{*}, r^{*}\right): \mathcal{U} \rightarrow U \times U$ such that $\left(s^{*}, r^{*}\right)(U) \in F^{*}(U)$ for all $U$. Whenever $F^{*}(U)$ is single valued, $F^{*}(U)=$ $\left\{\left(s^{*}, r^{*}\right)(U)\right\}$.

We next show that if the one imposes the extra condition on the extended solution $F$ that the induced reference point always lies in the boundary of the utility possibility set, then $F$ meets the standard axioms only if it coincides with $F^{*}$. The necessity part of the proof runs along the standard avenue. Take any $\left(s^{*}, r^{*}\right) \in F^{*}(T)$ and normalize the situation such that $s^{*}=-d^{*}=(1,1)=: \mathbf{1}$. Identify the smallest symmetric rectangle $V$ that contains $U$. Then, by EIIA, $(\mathbf{1},-\mathbf{1}) \in F(U)$. The crucial point is that only at $\left(s^{*}, r^{*}\right)$ one can find a symmetric rectangle whose sides parallel the tangents of $U$ at both $s$ and $r$. Thus the theorem uses Nash's key argument to determine $s^{*}$ and $r^{*}$ simultaneously.

[Figure 1]

\footnotetext{
${ }^{8}$ As pointed out by the referee, a drawback of the extended Nash solution is that it is not continuous (in the Hausdorff metric) in the general domain of problems. As we shall see, the same is true for any extended solution meeting the four axioms. This is due to not anchoring the solution via fixed disagreement point. However, various domain restrictions, e.g. strict convexity, would remove the problem.
} 
Lemma 1 Suppose extended solution $F$ has the property that $r \in \bar{U}$ for all $U \in \mathcal{U}$ and for all $(s, r) \in F$. Then it satisfies PAR, EINV, EIIA, and ESYM if and only if $F=F^{*}$.

Proof. Checking PAR, EINV, ESYM, and EIIA is routine.

We show that if $(s, r)$ satisfies PAR, EINV, ESYM, and EIIA then $F^{*}(T)=F(T)$, for all $T \in \mathcal{U}$. First we argue that $F^{*}(T) \subset F(T)$.

Step 1: Take any $\left(s^{*}, r^{*}\right) \in F^{*}(T)$. Construct $U=a T+b$ such that

$$
a_{i}:=\frac{2}{s_{i}^{*}-r_{i}^{*}}, b_{i}:=-\frac{s_{i}^{*}+r_{i}^{*}}{s_{i}^{*}-r_{i}^{*}}, \text { for } i=1,2 .
$$

Then,

$$
a\left(s^{*}, r^{*}\right)+b=(\mathbf{1},-\mathbf{1}),
$$

for $\mathbf{1}:=(1,1)$. By EINV, $\left(s^{*}, r^{*}\right) \in F(T)$ if and only if $a\left(s^{*}, r^{*}\right)+b \in F(U)$.

Step 2: Identify rectangle $V$ such that $V=V^{\prime},\{\mathbf{1},-\mathbf{1}\} \subset \bar{V}$, and $U \subset V$ (see Fig. 1). By convexity of $U$, such $V$ exists.

Step 3: By ESYM, PAR, and property $r \in \bar{V}$ for all $(s, r) \in F,(\mathbf{1},-\mathbf{1}) \in$ $F(V)$. Since $U \subseteq V$ and $(\mathbf{1},-\mathbf{1}) \in U$, it follows by EIIA that $(\mathbf{1},-\mathbf{1}) \in F(U)$. Thus $a\left(s^{*}, r^{*}\right)+b \in F(U)$ and, a fortiori, $\left(s^{*}, r^{*}\right) \in F(T)$.

Finally, $F(T) \subset F^{*}(T)$ follows from $F^{*}(T) \subset F(T)$ and convexity of $F$.

Given Lemma 1, we only need to show that any admissible $F$ only induces reference points in the boundary of the utility possibility sets. To prove this, we first focus on a restricted domain.

Strictly Convex Problems Boundary of $U$ is smooth if it is at least tricely continuously differentiable. Define subdomain $\widetilde{\mathcal{U}}$ of $\mathcal{U}$ as follows:

$$
\widetilde{\mathcal{U}}=\{U \in \mathcal{U}: U \text { is strictly convex and has smooth boundary }\} .
$$

Note that the curvature of smooth $\bar{U}$ is also a continuous variable.

As discussed above, the only case where $\left(s^{*}, r^{*}\right)$ is not uniquely defined is when $U$ has parallel flat sides. Under $\widetilde{\mathcal{U}}$, this is not possible and hence $F^{*}=\left\{\left(s^{*}, r^{*}\right)\right\}$. The next corollary is an immediate consequence of Lemma 1 .

Corollary 2 Suppose extended solution $F$ has the property that $r \in \bar{U}$ for all $U \in \widetilde{\mathcal{U}}$ and for all $(s, r) \in F$. Then it satisfies PAR, EINV, ESYM, and EIIA on $\widetilde{\mathcal{U}}$ if and only if $F=\left\{\left(s^{*}, r^{*}\right)\right\}$.

The only complication is due to the nonexistence of the rectangular problem used in the proof of Lemma 1 in the domain under consideration. To verify the result one needs to come up with a symmetric problem in $\widetilde{\mathcal{U}}$ that 
serves in the role of the rectangular problem: it must contain $U \in \widetilde{\mathcal{U}}$ (such that $F^{*}(U)=\{(\mathbf{1},-\mathbf{1})\}$ ) and its boundary must contain points $\mathbf{1}$ and $\mathbf{- 1}$. There are many alternatives for such choice. However, it is beneficial to be explicit. Introduce a family of $45^{\circ}$-inclined ellipses:

$$
E_{h}:=\left\{u \in \mathbb{R}^{2}: \frac{\left(u_{1}-u_{2}\right)^{2}}{h^{2}}+\frac{\left(u_{1}+u_{2}\right)^{2}}{(2 \sqrt{2})^{2}} \leq \sqrt{2}\right\} .
$$

$E_{h}$ is an origin-centered ellipse and parametrized by the negative (with slope -1 ) diameter length $h$. The positive (with slope 1) diameter length of $E_{h}$ is always $2 \sqrt{2}$. Hence the boundary of $E_{h}$ contains points $\mathbf{1}$ and $\mathbf{- 1}$. Any $E_{h}$ is symmetric, strictly convex and smooth. Since $U$ is strictly convex, there are big enough $h^{\prime}$ and small enough $h^{\prime \prime}$ such that $E_{h^{\prime \prime}} \subset U \subset E_{h^{\prime}}$ (see Fig. $2)$.

[Figure 2]

Our next aim is to show that under domain $\widetilde{\mathcal{U}}$, restriction $r \in \bar{U}$ for all $U \in \mathcal{U}$ for all $(s, r) \in F$ is redundant - it is implied by the other four conditions. Thus PAR, EINV, ESYM, and EIIA completely characterize the extended Nash solution under $\widetilde{\mathcal{U}}$. This result is established in Theorem 5 .

Before proving the theorem, we need some intermediate arguments. Take $U \in \widetilde{\mathcal{U}}$. For any $r \in U$, identify the corresponding (standard) Nash solution $s^{*}(\cdot, r): \widetilde{\mathcal{U}} \rightarrow \mathbb{R}^{2}:$

$$
s^{*}(U, r):=\underset{u \in U}{\arg \max }\left(u_{1}-r_{1}\right)\left(u_{2}-r_{2}\right) .
$$

Disagreement point $r$ is fixed. Note that any $r$ induces a unique (single valued) Nash solution, and that $r$ 's inducing a particular solution lie in the same upward sloping line segment that also contains the solution. Since a straight line crosses the boundary of a strictly convex set at most two times, two distinct disagreement points in the boundary cannot induce the same solution. This fact is established formally in the next Lemma.

Lemma 3 If $r, q \in \bar{U}$ and $s^{*}(U, r)=s^{*}(U, q)$, then $r=q$.

Fix $U \in \widetilde{\mathcal{U}}$. Take any $q \in U$, and identify coefficients $(a(q, r), b(q, r)) \in$ $\mathbb{R}_{+}^{2} \times \mathbb{R}^{2}$ such that

$$
a_{i}(q, r):=\frac{r_{i}-s_{i}^{*}(U, r)}{q_{i}-s_{i}^{*}(U, q)}, b_{i}(q, r):=\frac{s_{i}^{*}(U, r) q_{i}-s_{i}^{*}(U, q) r_{i}}{q_{i}-s_{i}^{*}(U, q)}, \text { for all } i=1,2 .
$$


Then $a(q, r) s^{*}(U, q)+b(q, r)=s^{*}(U, r)$ and $a(q, r) q+b(q, r)=r$. Identify subset $Q(r)$ of $U$ such that

$$
Q(r):=\{q \in U: a(q, r) U+b(q, r) \subset U\} .
$$

Thus $Q(r)$ contains such $q$ of $U$ that if $U$ is normalized so that $q$ and the Nash solution induced by $q$ coincide with $r$ and the Nash solution induced by $r$, then the scaled version of $U$ is still contained in $U$. Note that $r \in Q(r)$.

We now argue that $Q(r) \cap \bar{U}$ is single valued only if $r \in \bar{U}$. Identify $\bar{r} \in \bar{U}$ that induces same Nash solution than $r$ does, and suppose that $\bar{r} \neq r$. Normalize $U$ such that scaled $\bar{r}$ coincides with original $r$, and such that the scaled $\bar{r}$-induced Nash solution coincides with the original one. Call the normalized $U$ by $U^{*}$. Since $r$ is not at the boundary, $U^{*}$ is a "smallscaled" version of $U$, and $U^{*} \subset U$. Thus $U^{*}$ can potentially be "shaked" slightly such that the Nash solution of the shaked problem coincides with the original solution, e.g. by replacing in the beginning $\bar{r}$ with some $q^{k} \in \bar{U}$ close to it, without crossing the exterior surface of $U$. Lemma 4 proves that this indeed can be done (see Figure 3a).

The result relies on the fact that the curvature of the boundary of the small-scaled solution is higher than the original problem. Smoothness of the boundary guarantees that there is a neighborhood around the Nash solution where the boundary of the shaked small-scaled version of $U$ does not cross the boundary of $U$.

Proof of Lemma 4 actually shows that any reference point $q^{k}$ in some neighborhood of $\bar{r}$ induces a normalization of $U$, say $U^{k}$, that is contained in $U$. Thus $Q(r)$ contains all the elements in this neighborhood. Potential $Q(r)$ is depicted in Figure 3b. Note that the $r$-induced Nash solution under $U^{k}$ is not the normalized $\bar{r}$-induced Nash solution of $U$.

[Figures $3 \mathrm{a}$ and $3 \mathrm{~b}$ ]

Lemma 4 Take $U$ and $r \notin \bar{U}$. Let $Q(r)$ be defined as in (4). Then $Q(r) \cap \bar{U}$ contains at least two distinct element.

Proof. Identify $\bar{r} \in \bar{U}$ such that $s^{*}(U, \bar{r})=s^{*}(U, r)$. By construction, $\bar{r} \in Q(r)$. Take any sequence $\left\{q^{k}\right\} \subset \bar{U}$ converging to $\bar{r}$ such that $\bar{r} \notin\left\{q^{k}\right\}$. Then, by (3),

$$
a_{i}\left(q^{k}, r\right) \rightarrow a_{i}(\bar{r}, r) \text { and } b_{i}\left(q^{k}, r\right) \rightarrow b_{i}(\bar{r}, r) \text {, as } k \rightarrow \infty \text {, for } i=1,2 .
$$


Define $U^{k}:=a\left(q^{k}, r\right) U+b\left(q^{k}, r\right)$ for all $k=1, \ldots$, and $U^{*}:=a(\bar{r}, r) U+b(\bar{r}, r)$. By (5), $\left\{U^{k}\right\}$ converges (in the Hausdorff metric) to $U^{*}$. By construction, $s^{*}\left(U^{k}, q^{k}\right)=s^{*}\left(U^{*}, r\right)$ for all $k$. Denote by $P, P^{*}$ and $P^{k}$ the Pareto frontiers of $U, U^{*}$ and $U^{k}$, respectively.

Without loss of generality, assume normalization $s^{*}(U, r)=\mathbf{0}$. Then $a_{i}(\bar{r}, r)=r_{i} / \bar{r}_{i}:=\rho<1$ and $b_{i}(\bar{r}, r)=0$ for all $i=1,2$. Thus

$$
U^{*}=\rho U \text {. }
$$

Since $U$ is strictly convex, $U^{*} \subset U$ and $\bar{U} \cap \bar{U}^{*}=\{\mathbf{0}\}$. By convergence of $\left\{U^{k}\right\}$, there is $k(u) \in \mathbb{N}$ such that $u \notin i n t U^{k}$ for all $k>k(u)$ for any $u \in \bar{U}$. If $\sup _{u \in \bar{U}} k(u) \leq k^{*}$, then $q^{k^{*}} \in Q(r)$.

We aim at showing that $\sup _{u \in \bar{U}} k(u)$ is finite. Take any open neighborhood $B$ around $\mathbf{0}$. Since $\left\{U^{k}\right\}$ converges to $U^{*}$, set $\{k(u): u \in \bar{U} \backslash B\}$ is necessarily finite. It thus suffices to show that there is neighborhood $B$ around $\mathbf{0}$ such that $\sup \{k(u): u \in \bar{U} \cap B\}$ is finite, or that $B \cap \bar{U} \cap \bar{U}^{k^{\prime}}=\{\mathbf{0}\}$ for all $k^{\prime}>k$, for some $k$.

Describe the Pareto frontiers of $U, U^{*}$ and $U^{k}$ by functions $\beta:\left\{u_{1}\right.$ : $u \in P\} \rightarrow\left\{u_{2}: u \in P\right\}, \beta_{*}:\left\{u_{1}: u \in P^{*}\right\} \rightarrow\left\{u_{2}: u \in P^{*}\right\}$ and $\beta_{k}:\left\{u_{1}: u \in P^{k}\right\} \rightarrow\left\{u_{2}: u \in P^{k}\right\}$, respectively. Since $U$ is smooth, $\beta^{\prime \prime}$ is continuous. Now, by normalization (6),

$$
\beta_{k}^{\prime \prime}(0)=\frac{a_{2}\left(q^{k}, r\right)}{a_{1}\left(q^{k}, r\right)} \beta_{*}^{\prime \prime}\left(\frac{b_{1}\left(q^{k}, r\right)}{a_{1}\left(q^{k}, r\right)}\right) \rightarrow \beta_{*}^{\prime \prime}(0), \text { as } k \rightarrow \infty,
$$

where the first equality is by definition, and the second by (5). On the other hand, by (6),

$$
\beta_{*}^{\prime \prime}(0)=\rho^{-1} \beta^{\prime \prime}(0) .
$$

Since $U$ is strictly convex and smooth,

$$
\beta^{\prime \prime}(0)<0 .
$$

Thus, by (7) and $\rho<1$, there is $k$ such that

$$
\beta_{k}^{\prime \prime}(0)<\beta^{\prime \prime}(0) \text {, for all } k^{\prime} \geq k \text {. }
$$

Let $\Delta(x):=\beta_{k}(x)-\beta(x)$. Assume $x>0$. Since $\beta_{k}(0)=\beta(0)(=0)$ and $\beta_{k}^{\prime}(0)=\beta^{\prime}(0)\left(=r_{2} / r_{1}\right)$, Taylor expansion at 0 yields

$$
\Delta(x)=\frac{x}{2}\left[\Delta^{\prime \prime}(x)+\frac{x}{3} \Delta^{\prime \prime \prime}\left(x^{\prime}\right)\right], \text { for some } x^{\prime} \in[0, x] .
$$

By smoothness of $U, \Delta^{\prime \prime \prime}$ is a continuous function. Thus $\Delta^{\prime \prime \prime}$ reaches its maximum on $[0, x]$. Since

$$
\max \left\{\Delta^{\prime \prime \prime}\left(x^{\prime}\right): x^{\prime} \in[0, x]\right\} \geq \max \left\{\Delta^{\prime \prime \prime}\left(x^{\prime}\right): x^{\prime} \in[0, y]\right\}, \text { for any } y \leq x,
$$


there exists $\varepsilon^{\prime}>0$ such that

$$
\Delta(x)<0 \text {, for all } x \in\left(0, \varepsilon^{\prime}\right) .
$$

Thus $\beta_{k}(x)<\beta(x)$ for all $x \in\left(0, \varepsilon^{\prime}\right)$.

Analogously, there is $\varepsilon^{\prime \prime}>0$ such that $\beta_{k}(x)<\beta(x)$ for all $x \in\left(-\varepsilon^{\prime \prime}, 0\right)$. By choosing $\varepsilon=\min \left\{\varepsilon^{\prime}, \varepsilon^{\prime \prime}\right\}$, there is an open $\varepsilon-$ ball $B_{\varepsilon}$ around $\mathbf{0}$ such that $B_{\varepsilon} \cap \bar{U} \cap \bar{U}^{k^{\prime}}=\{\mathbf{0}\}$ for all $k^{\prime}>k$, as desired.

The important implication of this result is that if $r$ does not lie in the boundary of $U$, for some $(s, r) \in F(U)$, then there are (i) several distinct reference points in the boundary such that (ii) when $U$ is scaled so that the induced solution and the reference point inducing the solution correspond $(s, r)$, then the small-scaled $U$ is contained by $U$. As a consequence, (ii) implies by EIIA that $(s, r)$ should also belong to the extended solution under scaled utilities. By (i) there are many scaled versions of $U$ that has to meet this property. By EINV and Lemma 4, $\{s:(s, r) \in F(U)\}$ has to contain many elements. But this violates convexity of the extended solution $F$. Thus $r$ has to lie in the boundary of $U$. This together with Corollary 2 implies that the four standard axioms alone characterize the extended solution.

Theorem 5 Extended solution F satisfies PAR, EINV, ESYM and EIIA on $\widetilde{\mathcal{U}}$ if and only if $F=\left\{\left(s^{*}, r^{*}\right)\right\}$. $\tilde{\mathcal{U}}$.

Proof. By construction, $F^{*}$ satisfies PAR, EINV, ESYM and EIIA on

To see the other direction, repeat Steps 1-2 of Theorem 1. To check the third step, note that by ESYM and PAR, $(s, r)=(\mathbf{1}, \rho \mathbf{1})$ for $\rho \in[-1,1)$, for some $(s, r) \in F\left(E_{h}\right)$. If $\rho=-1$, then $r \in \bar{U}$ and Theorem 1 applies. Thus suppose $\rho \in(1,-1)$. Since $U \subseteq E_{h}$ and $(\mathbf{1}, \rho \mathbf{1}) \in U$, and since $U$ is strictly convex, it follows by EIIA that $(s, r)=(\mathbf{1}, \rho \mathbf{1})$ for some $(s, r) \in F(U)$.

Since $\rho \mathbf{1} \notin \bar{U}$, it follows by Lemma 4 that $Q(\rho \mathbf{1}) \cap \bar{U}$ is nonempty. Take any $q \in Q(\rho \mathbf{1}) \cap \bar{U}$ and identify $U^{q}=a(q, \rho \mathbf{1}) U+b(q, \rho \mathbf{1})$. Then, by construction,

$$
\begin{aligned}
s^{*}\left(U^{q}, \rho \mathbf{1}\right) & =s^{*}(U, \rho \mathbf{1})=\mathbf{1}, \\
a(q, \rho \mathbf{1}) q+b(q, \rho \mathbf{1}) & =\rho \mathbf{1}, \text { and } \\
U^{q} & \subseteq E_{h} .
\end{aligned}
$$

By EIIA and convexity of $F$,

$$
s=\mathbf{1}, \text { for all }(s, r) \in F\left(U^{q}\right) .
$$

On the other hand, by EINV,

$$
s^{\prime}=a(q, \rho \mathbf{1}) \mathbf{1}+b(q, \rho \mathbf{1}), \text { for all }\left(s^{\prime} r^{\prime}\right) \in F\left(U^{q}\right) .
$$


By (3),

$$
\begin{aligned}
s_{i}^{\prime} & =\frac{\rho-s_{i}^{*}(U, \rho \mathbf{1})}{q_{i}-s_{i}^{*}(U, q)}+\frac{s_{i}^{*}(U, \rho \mathbf{1}) q_{i}-s_{i}^{*}(U, q) \rho}{q_{i}-s_{i}^{*}(U, q)} \\
& =\frac{q_{i}-1+\left(1-s_{i}^{*}(U, q)\right) \rho}{q_{i}-s_{i}^{*}(U, q)} .
\end{aligned}
$$

Combining (8) and (9),

$$
q_{i}-1+\left(1-s_{i}^{*}(U, q)\right) \rho=q_{i}-s_{i}^{*}(U, q) .
$$

Thus,

$$
s^{*}(U, q)=\mathbf{1} .
$$

By Lemma $4, Q(\rho \mathbf{1}) \cap \bar{U} \backslash\{q\}$ is nonempty. Since $q$ was chosen arbitrarily, $s^{*}\left(U, q^{\prime}\right)=\mathbf{1}$ for all $q^{\prime} \in Q(\rho \mathbf{1}) \cap \bar{U} \backslash\{q\}$. But this contradicts Lemma 3 .

The Main Result But we can say more. Lemma 1 is dependent on the restriction that any symmetric rectangular problem $V$ induces symmetric $(s, r) \in F(V)$ such that $r \in \bar{V}$, and such that $\mathbf{1},-\mathbf{1} \in \bar{V}$. We argue that also this restriction is redundant: it is implied by the other conditions stated in the theorem. This in turn implies that the four standard axioms characterize the extended Nash solution even in the general domain. This is the main result of the paper.

To see why the restriction is redundant, note that Theorem 5 implies that any extended solution $F$ meeting PAR, EINV, ESYM and EIIA satisfies $F(U)=F^{*}(U)$ for all $U \in \widetilde{\mathcal{U}}$. Assume now that $r \notin \bar{V}$ for symmetric $(s, r) \in F(V)$. Invoke $U \in \widetilde{\mathcal{U}}$ that on the one hand contains $\mathbf{1}$ and $\mathbf{- 1}$ and, on the other, is contained by $V$. For example, small enough $E_{h}$ would do (see Fig. 4). Then, by EIIA, $(s, r) \in F(U)$. But this is in contradiction with $F(U)=F^{*}(U)$. Thus $r \in \bar{V}$ for symmetric $(s, r) \in F(V)$.

[Figure 4]

Theorem 6 (Main) Extended solution F satisfies PAR, EINV, ESYM, and EIIA on $\mathcal{U}$ if and only if $F=F^{*}$.

Proof. By Lemma 1, it suffices to show that PAR, EINV, ESYM, and EIIA imply that $r \in \bar{V}$ for symmetric $(s, r) \in F(V)$, for all symmetric rectangular problems $V \in \mathcal{U}$. Without loss, assume that boundary of $V$ contains points $\mathbf{1}$ and $\mathbf{- 1}$ and that its side length is $h$. Then $E_{h} \subset V$. Suppose that $r \neq-\mathbf{1}$ for symmetric $(s, r) \in \bar{V}$. By EIIA, $(s, r) \in F\left(E_{h}\right)$. Since $E_{h} \in \widetilde{\mathcal{U}}$, Theorem 5 implies that $\left(s^{\prime}, r^{\prime}\right) \in F\left(E_{h}\right)$ only if $\left\{\left(s^{\prime}, r^{\prime}\right)\right\}=F^{*}\left(E_{h}\right)$. But this implies $r=\mathbf{- 1}$, a contradiction 


\section{Alternative Interpretation}

Admittedly, the interpretation of a reference point is not completely compelling. Why is it that the players are interested in a single reference point rather than many? To this end we provide an alternative motivation for the current approach that is based on more reasonable groundings. We argue that the solution correspondence $s^{*}(U):=\left\{s:(s, r) \in F^{*}(U)\right\}$ for $U \in \mathcal{U}$, can be motivated through many other constructions of of the extended solution concept.

To be more precise, define a generalized solution correspondence $\mathcal{G}$ on $\mathcal{U}$ such that $\mathcal{G}(U)$ consists of collection of triples $(s, G, \succ)$, where $G$ is a nonsingleton, compact reference set such that $G \subseteq U, \succ$ is a complete and transitive partial order ("collective preference order") on $G$, and $s$ is a solution such that $s:=\max _{\succ} G$. ${ }^{9}$ Since $G$ is nonsingleton, there is $u \neq s$ such that $u \in G$. Assume that $\mathcal{G}(U)$ is convex in a sense that $(s, G, \succ),\left(s^{\prime}, G^{\prime} \succ^{\prime}\right) \in \mathcal{G}(U)$ imply $\left(s \lambda+s^{\prime}(1-\lambda), G^{\prime \prime}, \succ^{\prime \prime}\right) \in \mathcal{G}(U)$ for some $\left(G^{\prime \prime}, \succ^{\prime \prime}\right)$ such that $s \lambda+s^{\prime}(1-\lambda)=\max _{\succ}{ }^{\prime \prime} G^{\prime \prime}$.

The interpretation of $G$ is now that players bargain by eliminating outcomes from the set of potential decisions, and make the decision once the remaining set of outcomes, $G$, can be completely ordered according to some collective preference criterion $\succ$. Once such stage is reached in the bargaining process, it is easy to pick up the collectively most preferred outcome $s$. Hence if one does not want to artificially restrict the extended solution to contain only two elements, the generalized solution allows a larger set of reference alternatives.

The generalized versions of the axioms that govern the selection of the solution correspondence are written as follows.

- Generalized Pareto-optimality: If $(s, G, \succ) \in \mathcal{G}(U)$, then $s$ is Paretooptimal.

Pair $\left(G^{\prime}, \succ^{\prime}\right)$ is a transpose of $(G, \succ)$ iff $G^{\prime}:=\left\{\left(u_{2}, u_{1}\right):\left(u_{1}, u_{2}\right) \in\right.$ $G\}$ and $\left(u_{1}, u_{2}\right) \succ\left(v_{1}, v_{2}\right)$ whenever $\left(u_{2}, u_{1}\right) \succ^{\prime}\left(v_{2}, v_{1}\right)$, for all $u, v \in G$. Now $(G, \succ)$ is symmetric if and only if it is equal to its transpose. As a consequence, if $(G, \succ)$ is symmetric, then $(s, G, \succ)$ is symmetric.

- Generalized Symmetry: If $U$ is a symmetric problem, then $\mathcal{G}(U)$ contains a symmetric element.

Note that symmetry of $(s, G, \succ)$ implies that $G$ is contained by the diagonal (with slope 1) of $U$. Thus if $G$ contains more than two elements, then there is $u \in G \backslash \bar{U}$.

\footnotetext{
${ }^{9}$ Existence of such point is assumed.
} 
- Generalized Independence of Irrelevant Alternatives (IIA): If $(s, G, \succ) \in$ $\mathcal{G}(V), s, u \in G \cap U$ for some $u \neq s$, and $U \subseteq V$, then $(s, G \cap U, \succ) \in$ $\mathcal{G}(U)$.

Generalized IIA now reflects the idea that if $u$ is collectively preferred over $v$ under $V$, and $u, v \in U \subseteq V$, then $u$ must be collectively preferred over $v$ under $V$ as long as the preference ordering that is to be constructed leads to the same choice under $U$ and $V$. Thus the forces that govern elimination process under $V$ are also relevant under $U \subseteq V$, and those parts of $G$ that can be ranked according to $\succ$ under $V$ are also rankable under $U$ as long as they induce the same optimal choice. Note that the generalized IIA only binds when $(G \cap U) \backslash\{s\}$ contains an element.

- Generalized Scale Invariance: $\mathcal{G}$ is invariant to utility scales.

Note that an extended solution $F$ induces a generalized solution $\mathcal{G}^{F}$ such that

$$
\mathcal{G}^{F}(U):=\{(s,\{s, r\}, \succ):(s, r) \in F(U) \text { and } s \succ r\}, \text { for all } U \in \mathcal{U} .
$$

Thus there is also a natural way to render $F^{*}$ into a generalized solution $\mathcal{G}^{F^{*}}$.

It is important to note that the family of generalized solutions is much larger than those induced by extended solutions. Nevertheless, it is now almost routine to check that a generalized solution satisfies the generalized axioms if and only if it coincides with $\mathcal{G}^{F^{*}}$.

The proof of necessity, which is the more difficult part, proceeds as in the previous section. First use the argument of Lemma 1. Prove that note that for any appropriately scaled $U$ there is symmetric $V$ that contains $U$, and that induces $(s, G, \succ)$ where $G$ is, by generalized symmetry, contained by the positive diagonal of $V$, and $G \subset U$. Thus by generalized IIA, $(s, G, \succ) \in$ $\mathcal{G}(U)$. Moreover, if there is $r \neq s$ such that $r \in G \cap \bar{U}$, then $(s, r) \in F^{*}(U)$. To prove that $G$ contains only $\{s, r\}$ and $r \in \bar{U}$, for $U \in \widetilde{\mathcal{U}}$, assume this is not the case. Then there is $u \in G$ such that $u \notin \bar{U}$. Replace $r$ with $u$ and and construct $Q(u)$ as in definition (4). As in Theorem 5, use the argument of Lemma 4 together with convexity of $\mathcal{G}(U)$ to prove that $u \notin \bar{U}$ cannot be the case. Thus $G$ contains only two elements (but not less since $G \backslash\{s\}$ is nonempty), and both of them lie in the boundary. Then it follows that $(s, G, \succ) \in \mathcal{G}(U)$ only if $G=\{s, r\}$ such that $(s, r) \in F^{*}(U)$, and $s \succ r$. Finally, use Theorem 6 to prove the same thing in the unrestricted domain.

Now solution $F^{*}$ can be thought as an expression of the generalized solution that meets the generalized axioms. It is clear that the derivation of $F^{*}$ already contains the critical arguments needed to characterize $\mathcal{G}^{F^{*}}$. In fact, extended solution $F^{*}$ can be seen as the reduced form expression of $\mathcal{G}^{F^{*}}$ that already contains the argument that $G=\{s, r\}$. 
Theorem 7 Generalized solution $\mathcal{G}$ satisfies the generalized Pareto optimality, symmetry, IIA and scale invariance axioms if and only if $\mathcal{G}=\mathcal{G}^{F^{*}}$.

\section{Concluding Remarks}

In this paper we have argued the standard bargaining framework can be extended in a natural way to allow axiomatically endogenous formation of the reference point. We have shown that the extended solution, which determines endogenously both the solution and the reference point, is uniquely characterized by the appropriately adjusted Nash-axioms. The result is surprising since the formation of the disagreement point is completely characterized through the axioms.

As the solution to the current problem is derived as a function of preferences (which are reflected by the utility possibility set) alone, framework at hand belongs to the realm of social choice. The key difference to the standard social choice analysis is the fact that we employ an adjucant reference point to measure distancies from the solution. These distances are then used as the primitive to derive the solution. The approach allows us to circumvent the usual impossibility reults in the social choice literature.

In the light of Conley at.al. (1997, forth.), need for the adjucant reference point should not be surprising. Indeed they point out that the central difference between social choice and bargaining literatures concerns the existence of a reference or disagreement point from which utility comparisons can be made. In the former case, the nonexistence of a reference point leads to impossibility results whereas in latter case the existence of a reference point allows permissive results. More generally Conley at.al. show that any scale invariant from which to measure relative utility gains permits one to use the comparison axioms (such as IIA) and, a fortiori, to derive possibility results. Following Thomson (1981), they also characterize conditions for reference functions on the domain of utility possibility sets that allow one to derive unique solution with respect to the defined reference function. Thus the induced solution is dependent on the axioms that define it, and on the reference function. Since there are many reference functions, a family of solutions is induced for any given axiomatization. However, no attempt is made towards comparing the reference functions. Conley at. al. leave open the question which are the most desirable properties of a reference function.

The question of moving disagreement point has not gone without recognizion in the literature. In the literature, the approach has typically been to demand solution to be robust against certain positional changes of the disagreement point. Such condition can then be used in the characterization of solutions. Recently, Dagan at. al. (2002) show that IIA can be replaced with three conditions, independence of non-individually rational alternatives (INIR), twisting, which is a monotonicity requirement, and 
disagreement point convexity (DPC) in the characterization of the Nash solution. Chun and Thomson (1990) replace IIA with INIR, DPC and a continuity condition. Peters and van Damme (1991) assume INRA, individual rationality, DPC and a starshaped inverse condition (in the language of Thomson, 1991). However, this literature does not address the question of how should the disagreement point be chosen which is the central theme of this paper. To the authors knowledge, the notion of extended Nash solution is novel in the literature.

In an interesting contribution, Mariotti (1999) introduces a maximal symmetry axiom which, in spirit, has connections to our construction. Maximal symmetry requires that a solution with relabeled player names should belong to the original utility space. Marriotti shows that maximal symmetry and strong individual rationality can be used to replace IIA in the Nash characterization. Large domain of utility sets is needed. Thus the Nash solution is characterized through the extended usage of the symmetry properties of the solution. Extensive use of the symmetry properties of the extended Nash solution is also the driving factor of our model. This property is more transparently represented through the axiomatization of Vartiainen (2003), the old version of this paper.

\section{References}

[1] Chun, Y. And Thomson, W. (1990), Nash Solution and Uncertain Disagreement Points, Games and Economic Behavior 2, 213-23.

[2] Conley, J., Mclean R., And Wilkie S. (1997), Reference Functions and Possibility Theorems for Cardinal Social Choice Theory, Social Choice and Welfare, Vol. 14, 1997, pp. 65-78.

[3] Conley, J., Mclean R., And Wilkie S., Axiomatic Foundations for Compromise Theory: The Duality of Bargaining Theory and MultiObjective Programming, Games and Economic Behavior, forthcoming.

[4] Dagan N., Volij, O., And Winter E. (2002), A Characterization of the Nash Bargaining Solution, manuscript.

[5] Kalai, E. (1985), Solutions to the Bargaining Problem, in Hurwicz, Schmeidler and Sonnenschein (eds.), Social Goals and Social Organization, 77-105, Cambridge Univ. Press.

[6] Kalai, E. and Smorodinsky, R. (1975), Other Solutions to Nash's Bargaining Problem, Econometrica 43.

[7] Kalai, E. and Rosenthal, R. (1978), Arbitration of Two-Party Disputes under Ignorance, Int. Journal of Game Theory 7, 65-72. 
[8] Myerson, R. (1978), Linearity, Concavity and Scale Invariance in Social Choice Functions, Northwestern university DP 321, Evanston, IL.

[9] Mariotti, M. (2000), Maximal symmetry and the Nash solution, Social Choice and Welfare 17, 45-53.

[10] Nash, J. (1950), The Bargaining Problem, Econometrica 28.

[11] Peters, H., and van Damme, E. (1991), Characterizing the Nash and Raiffa Solutions by Disagreement Point Axioms, Mathematics of Operations Research 16, 447-61.

[12] SEn, A. (1982), Choice, Welfare and Measurement, Harvard UP, Cambridge MA

[13] Thomson, W . (1981), A Class of Solutions to the Bargaining Problem, Journal of Economic Theory 25, 431-42.

[14] Thomson, W. (1994), Cooperative Models of Bargaining, Handbook of Game Theory, ch 35.

[15] Vartiainen, H. (2003), Bargaining without Disagreement, University of Helsinki DP 556. 


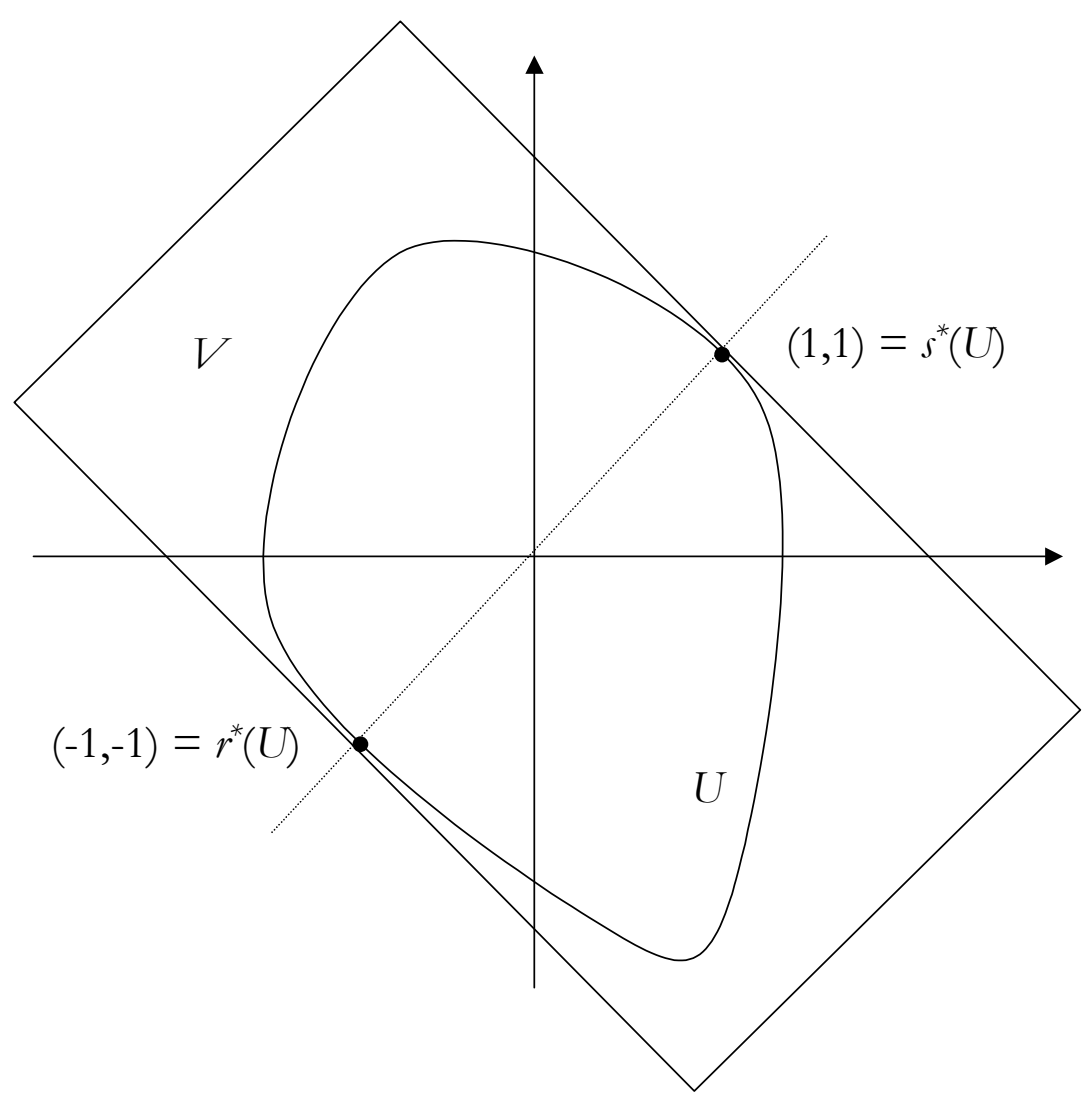

Figure 1. 


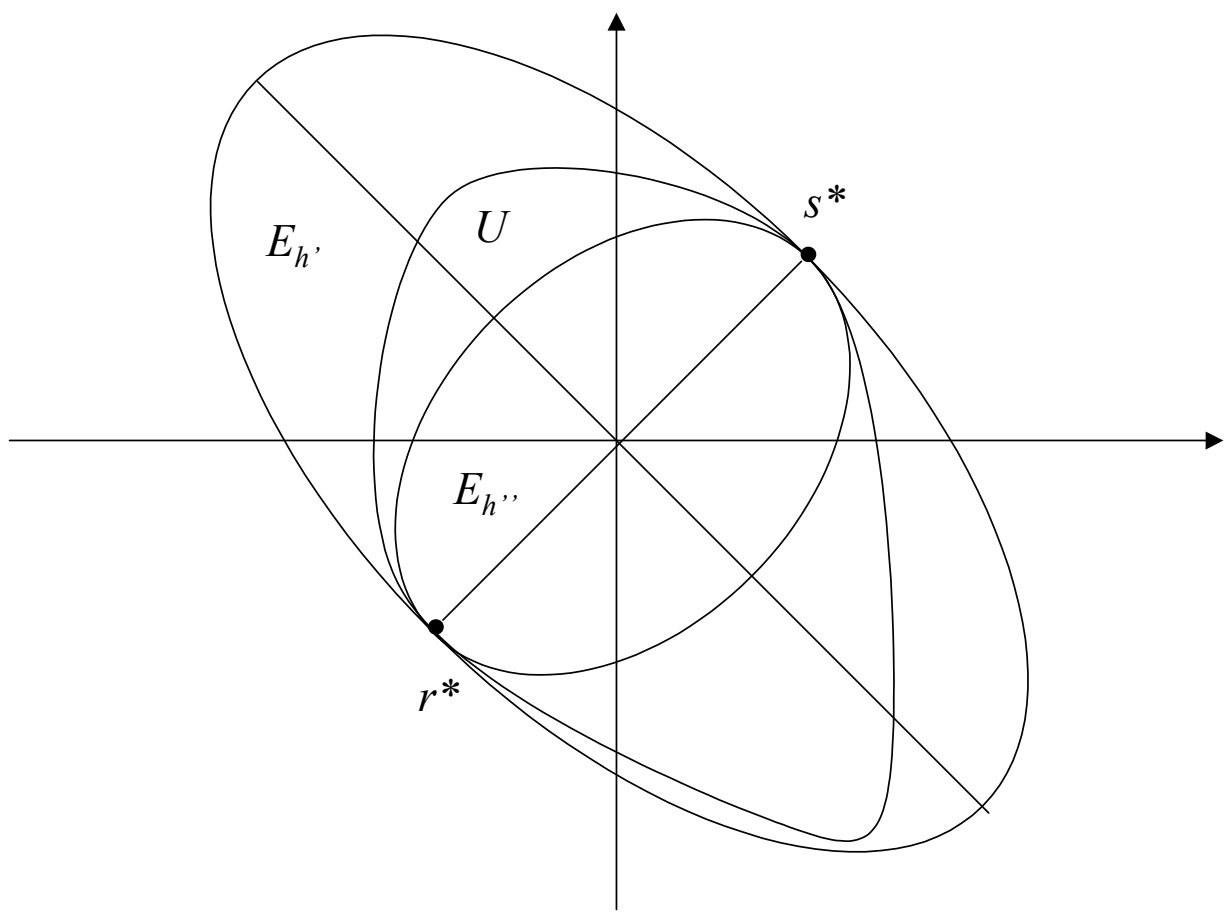

Figure 2 


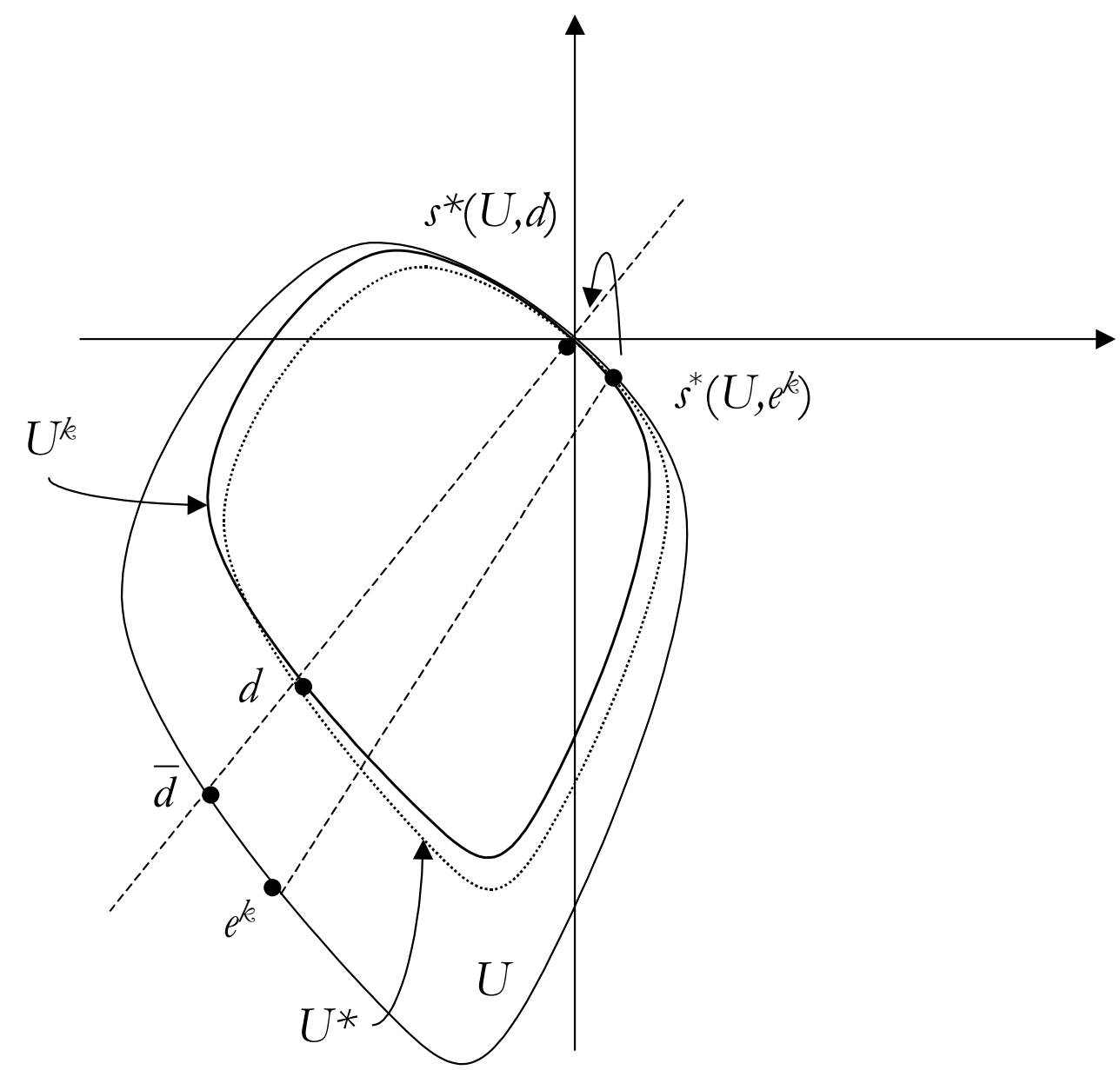

Figure $3 a$.

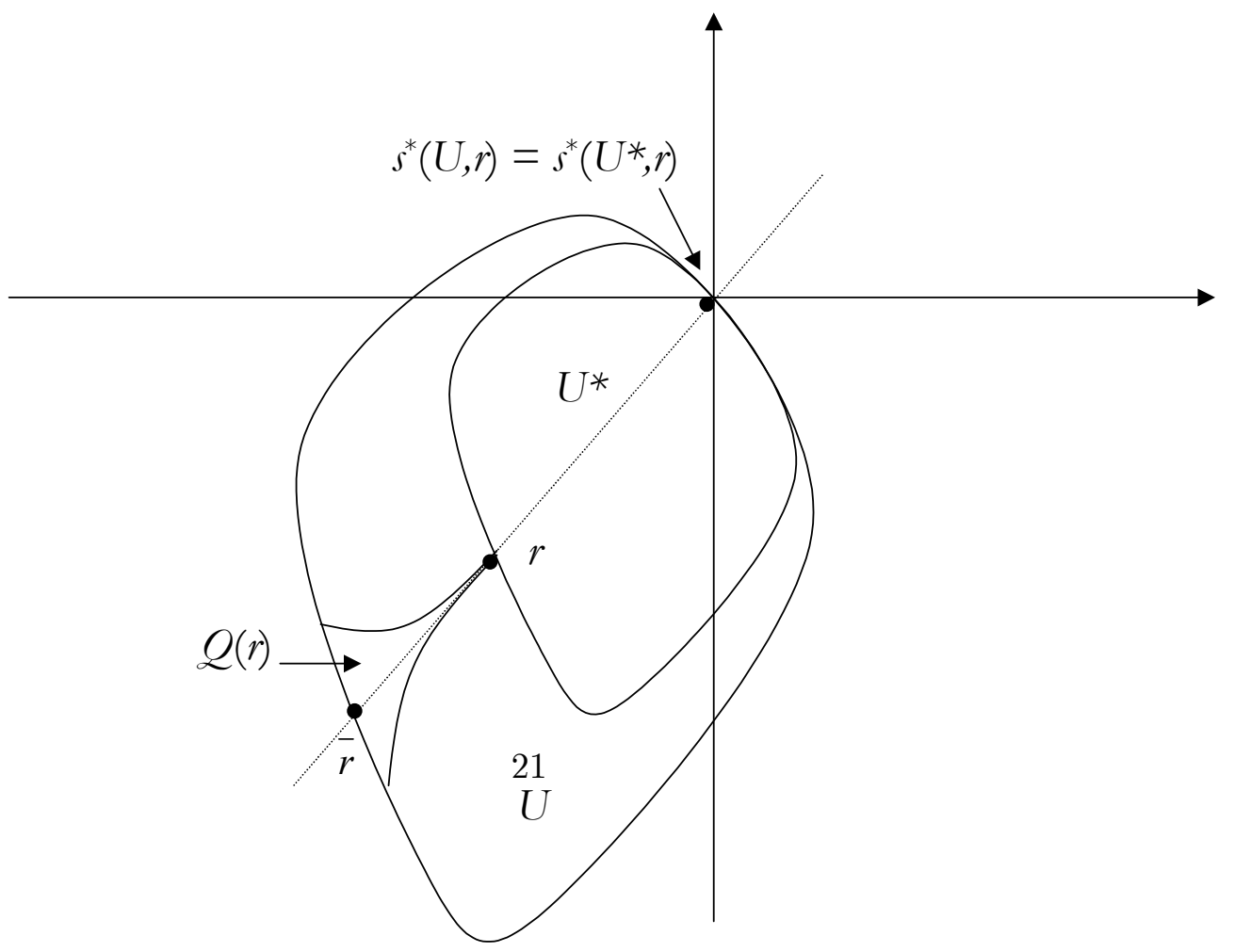

Figure $3 b$ 


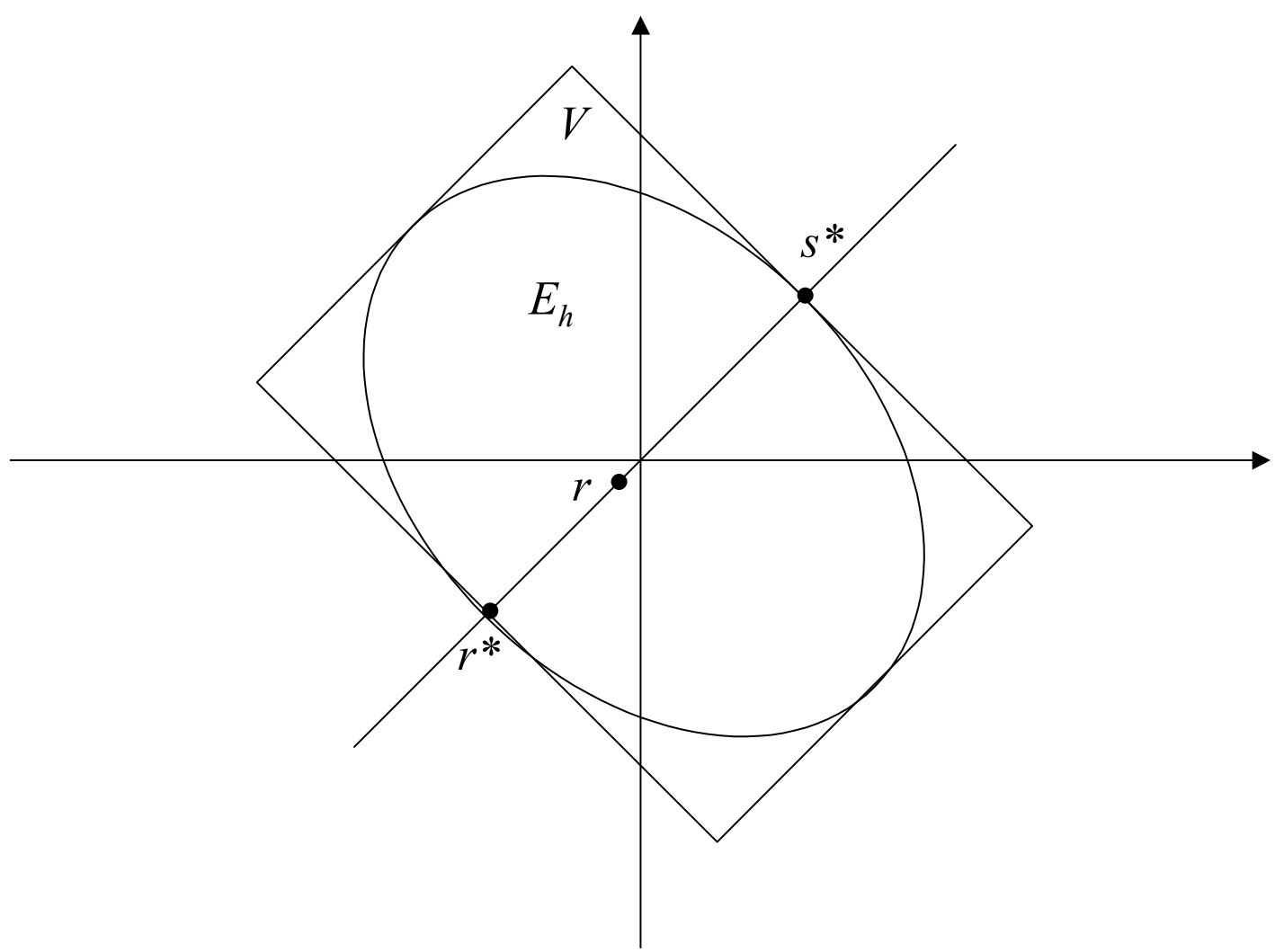

Figure 4. 